\title{
Timoma epitelial em um felino - Relato de caso
}

\author{
Epithelial thymoma in a feline - Case report \\ Timoma epitelial en un felino - Reporte de caso
}

\section{Resumo}

O objetivo do presente trabalho foi relatar um caso de timoma epitelial em um felino, encaminhado para diagnóstico no Laboratório Regional de Diagnóstico, da Faculdade de Veterinária, da Universidade Federal de Pelotas (LRD/FV/UFPel). Segundo o médico veterinário clínico responsável o animal apresentou histórico de vômito e hiporexia, evoluindo rapidamente para o óbito, sendo o cadáver encaminhado ao LRD/FV/UFPel. Na necropsia observou-se uma massa pardo-clara, lobulada e aparentemente encapsulada no mediastino anterior. Fragmentos da massa mediastínica e dos órgãos das cavidades abdominal e torácica, bem como, o encéfalo foram coletados e fixados em formalina 10\% tamponada. Após 48 horas as amostras de tecido foram clivadas, processadas rotineiramente e coradas pela técnica de hematoxilina e eosina. Cortes selecionados da massa mediastínica foram colhidos em lâmina positivada para realização da técnica de imuno-histoquímica (IHQ). Os anticorpos utilizados na IHQ foram o anticitoqueratina, CD3 e CD79a. Na avaliação histopatológica da massa mediastínica observou-se proliferação de células epiteliais do timo em meio a linfócitos não neoplásicos. Na técnica de IHQ as células neoplásicas foram positivas para citoqueratina. O diagnóstico de timoma epitelial, neste caso, foi baseado nos achados macroscópicos, microscópicos e confirmado pela imuno-histoquímica.

Palavras-chave: Medicina de felinos; Oncologia felina; Neoplasias torácicas; Timo.

\begin{abstract}
The aim of the present study was to report a case of epithelial thymoma in a feline, referred for diagnosis at the Regional Diagnostic Laboratory, Faculty of Veterinary Medicine, Federal University of Pelotas (LRD/FV/UFPel). According to the responsible clinical veterinarian, the animal presented a history of vomiting and hyporexia, rapidly progressing to death, and the cadaver was sent to the LRD/FV/UFPel. At necropsy, a light brown mass was observed, lobulated and apparently encapsulated in the anterior mediastinum. Fragments of the mediastinal mass and organs of the abdominal and thoracic cavities, as well as the brain were collected and fixed in $10 \%$ buffered formalin. After 48 hours the tissue samples were cleaved, routinely processed and stained using the hematoxylin and eosin technique. Selected sections of the mediastinal mass were collected on a positive slide to perform the immunohistochemical technique (IHC). The antibodies used in IHC were anti-cytokeratin, CD3 and CD79a. In the histopathological evaluation of the mediastinal mass, proliferation of thymic epithelial cells was observed among non-neoplastic lymphocytes. In the IHC technique, neoplastic cells were positive for cytokeratin. The diagnosis of epithelial thymoma in this case was based on macroscopic and microscopic findings and confirmed by immunohistochemistry.

Keywords: Feline medicine; Feline oncology; Thoracic neoplasms; Thymus.
\end{abstract}




\begin{abstract}
Resumen
El objetivo de este estudio fue reportar un caso de timoma epitelial en un felino, remitido para diagnóstico en el Laboratorio Regional de Diagnóstico, Facultad de Medicina Veterinaria, Universidad Federal de Pelotas (LRD / FV / UFPel). Según el veterinario clínico responsable, el animal presentó antecedente de vómitos e hiporexia, que progresó rápidamente hasta la muerte, y el cadáver fue enviado al LRD / FV / UFPel. En la necropsia se observó una masa de color marrón claro, lobulada y aparentemente encapsulada en el mediastino anterior. Se recogieron fragmentos de la masa mediastínica y órganos de las cavidades abdominal y torácica, así como del cerebro y se fijaron en formalina tamponada al 10\%. Después de 48 horas, las muestras de tejido se escindieron, se procesaron de forma rutinaria y se tiñeron utilizando la técnica de hematoxilina y eosina. Se recogieron secciones seleccionadas de la masa mediastínica en un portaobjetos positivo para realizar la técnica inmunohistoquímica (IHC). Los anticuerpos utilizados en IHC fueron anti-citoqueratina, CD3 y CD79a. En la evaluación histopatológica de la masa mediastínica se observó proliferación de células epiteliales tímicas entre linfocitos no neoplásicos. En la técnica IHC, las células neoplásicas fueron positivas para citoqueratina. El diagnóstico de timoma epitelial en este caso se basó en hallazgos macroscópicos y microscópicos y se confirmó por inmunohistoquímica.
\end{abstract}

Palabras clave: Medicina felina; Oncología felina; Neoplasias torácicas; Timo.

\title{
1. Introdução
}

O timo é um órgão linfóide primário, localizado no mediastino anterior e responsável pela maturação de linfócitos T em animais jovens. Em adultos o timo sofre um processo de regressão, sendo substituído por tecido adiposo (Freitas et al., 2002). Timomas são neoplasias de origem tímica, comumente benignas (Ortemberg et al., 2015). Em caninos e felinos ocorrem principalmente em animais com nove e dez anos de idade, sendo os sinais clínicos inespecíficos e relacionados com a localização do tumor (Day, 1997; Patnaik et al., 2003; Angelou et al., 2016).

Macroscopicamente apresentam-se como nódulos ovoides, encapsulados, branco-acinzentados de consistência variável, podendo apresentar áreas de aspecto necrótico. Microscopicamente são classificados como epiteliais, linfocíticos e/ou linfoepiteliais (mistos), de acordo com o padrão celular predominante no processo tumoral (Bandarra et al., 2000; Valli et al., 2017, Byas et al., 2019). A utilização de IHQ auxilia na determinação dos diferentes tipos histológicos de timoma (Patnaik et al., 2003; Capelozzi, 2009). Metástases são raras e quando presentes são observadas principalmente em pulmões e linfonodos mediastínicos (Moffet, 2007; Angelou et al., 2016).

Diferentes neoplasias podem, também, apresentar localização mediastínica, devendo ser incluídas como diagnóstico diferencial de timomas, sendo principalmente os linfomas, lipomas, lipossarcomas, teratomas, tumores ectópicos da tireóide e as metástases mediastínicas (Bandarra et al., 2000; Yoon et al., 2004; Horta et al., 2018). O diagnóstico diferencial é realizado através do exame citológico e histopatológico, sendo a imuno-histoquímico fundamental para determinar o subtipo de timoma.

O objetivo do presente trabalho foi relatar um caso de timoma epitelial em um felino, diagnosticado no Laboratório Regional de Diagnóstico da Faculdade de Veterinária da Universidade Federal de Pelotas (LRD/FV/UFPel).

\section{Metodologia}

Foi encaminhado ao LRD/FV/UFPel, o cadáver de um felino, fêmea, sem raça definida, de dez anos de idade, com histórico clínico de apatia, anorexia, vômito e emagrecimento progressivo. Durante a necropsia foram coletados e fixados em formalina 10\% tamponada, fragmentos dos órgãos das cavidades abdominal e torácica, bem como, encéfalo e fragmentos de uma massa localizada no mediastino anterior. Após 48 horas os fragmentos de tecido foram processados rotineiramente, incluídos em parafina, cortados em seç̧ões de $3 \mu \mathrm{m}$ de espessura e corados pela técnica de Hematoxilina e Eosina (HE). Cortes selecionados da massa mediastínica foram colhidos em lâminas positivadas e submetidos a técnica de imunohistoquímica (IHQ). Para a realização da IHQ utilizou-se anticorpo monoclonal primário anti-citoqueratina (clone AE1/AE3) na diluição de 1:100, anticorpo policlonal primário anti-CD3 na diluição de 1:200 e anti-CD79a na diluição de 1:400. As imunomarcações foram visualizadas com 3-3' diaminabenzidina (DAB). As secções foram contracoradas com Hematoxilina de 
Harris. Como controle negativo foram utilizadas secções do tecido incubadas apenas com o diluente do anticorpo.

\section{Resultados e Discussão}

$\mathrm{Na}$ necropsia do felino observou-se no mediastino anterior uma massa parda, lobulada e encapsulada, medindo 4,7x3,0x1,5cm (Figura 1A e 1B). Nos demais órgãos não foram observadas alterações macroscópicas de significado patológico.

Figura 1. Timoma em felino. 1A - Massa acima do coração e entre os pulmões (seta). 1B - Massa mediastínica pardo clara, lobulada e encapsulada, após a fixação em formalina 10\% tamponada.
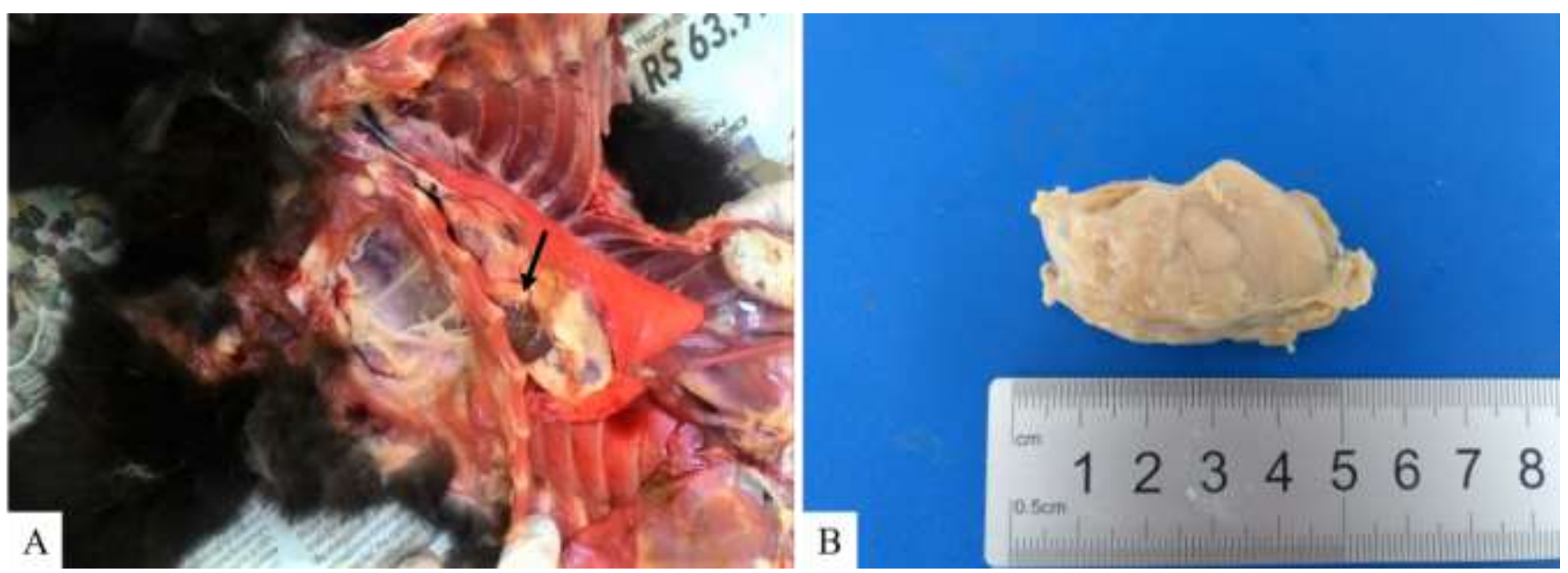

Fonte: Autores.

$\mathrm{Na}$ avaliação microscópica da massa mediastínica observou-se proliferação de células epiteliais bem diferenciadas, com núcleos arredondados a ovais e abundante citoplasma eosinofílico não delimitado (Figura 2A). As células estavam arranjadas em trabéculas e, por vezes, em um padrão sólido. Em meio as células havia linfócitos não neoplásicos e extensas áreas de necrose, com fendas de colesterol. Na avaliação imuno-histoquímica, houve imunomarcação positiva das células neoplásicas para citoqueratina (Figura 2B). 
Figura 2. Análise microscópica de timoma em felino. 2A - proliferação de células epiteliais tímicas. HE, Objetiva 4x. 2B Células epiteliais neoplásicas demonstrando imunomarcação positiva para citoqueratina. Objetiva 40x.
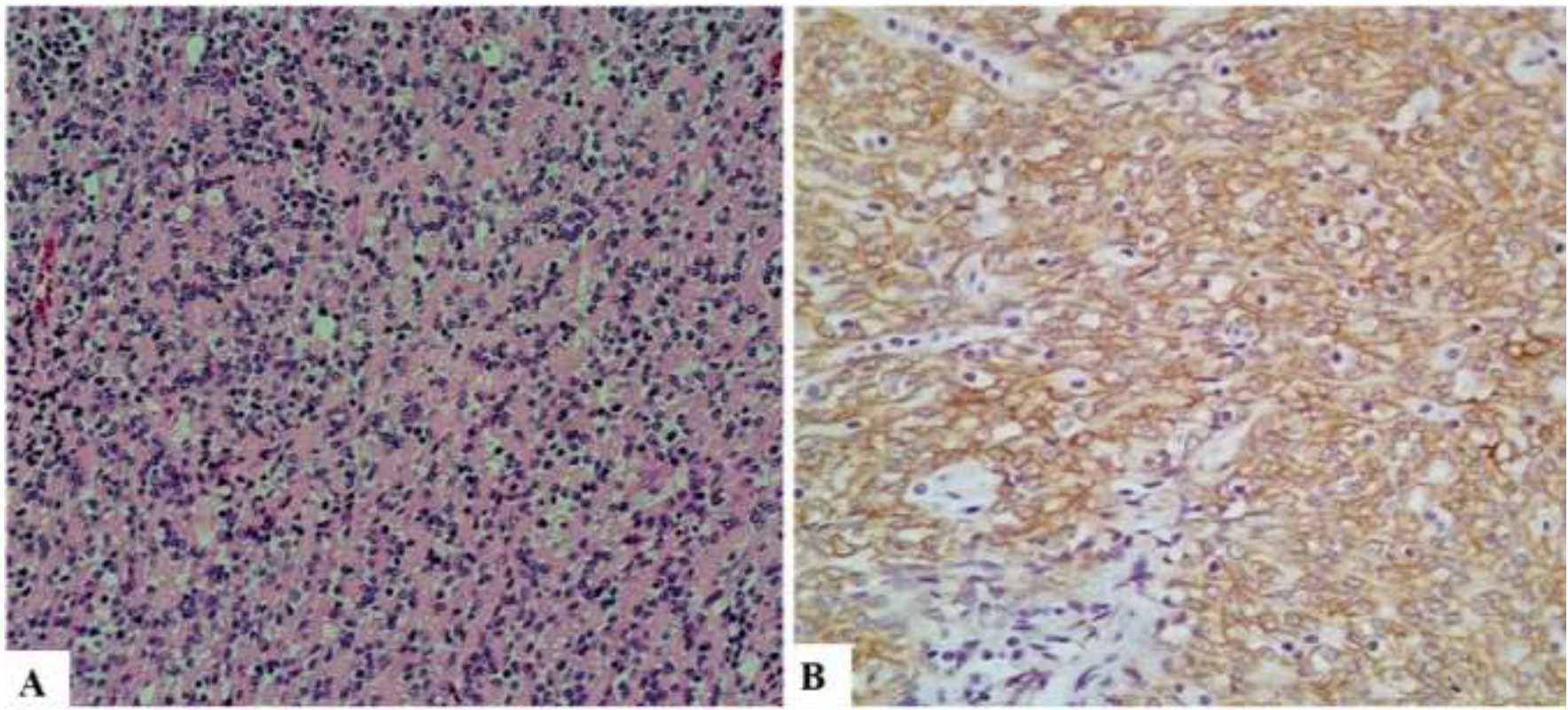

Fonte: Autores.

Em casos de neoplasias mediastínicas, como timomas, a realização de exames complementares como radiografias, ultrassonografias e tomografia computadorizada do tórax auxilia na detecção do neoplasma, porém o diagnóstico definitivo deve ser realizado através de exames citológicos e/ou histopatológico (Oliveira et al., 2021). No caso descrito devido a evolução rápida do quadro para o óbito do animal, não foram realizados exames de imagem, sendo o diagnóstico estabelecido pelos achados macroscópicos, microscópicos e imuno-histoquímico (IHQ).

Segundo a literatura, embora o exame histopatológico seja a técnica padrão para determinar o diagnóstico de timoma as características histológicas não são suficientes para determinar o grau de malignidade do neoplasma (Detterbeck et al., 2004; Horta et al., 2018), devendo sempre serem associadas com as características clínicas de invasividade e possibilidade de ressecção cirúrgica no momento do diagnóstico (Horta et al., 2018). No presente caso o timoma foi classificado histologicamente como grau I, porém, apesar desta classificação o animal evoluiu rapidamente para o óbito, corroborando com a literatura.

Nos casos de timoma deve ser realizado o diagnóstico diferencial de outras neoplasias mediastínicas, sendo o linfoma mediastínico o principal diferencial na espécie felina (Angelou et al., 2016). Em gatos os timomas ocorrem principalmente em animais adultos a idosos, com idade entre 9 e 10 anos e não apresenta relação com a infecção pelo vírus da leucemia felina (FeLV) (Souza et al., 2005; Valli et al., 2017), assim como no caso relatado. Já os linfomas ocorrem na maioria dos casos em animas jovens, FeLV positivos (Souza et al., 2005; Valli et al., 2017). Mediante a presença de neoplasia mediastínica é fundamental estabelecer o diagnóstico diferencial, visto que o tratamento de eleição varia de acordo com o tipo de neoplasia (Souza et al., 2005; Zitz et al., 2008; Angelou et al., 2016).

Os timomas são na maioria dos casos tumores benignos de evolução lenta, o que dificulta um diagnóstico precoce. Os sinais clínicos são inespecíficos e estão associados principalmente com a localização do tumor, sendo dispneia inspiratória, vômito, regurgitação ou disfagia os sinais clínicos mais frequentes, devido a compressão traqueal e esofágica respectivamente (Patnaik et al., 2003; Souza et al., 2005). No caso relatado, apesar do tamanho da massa tumoral o animal não apresentou sinais de dificuldade respiratória, salientando-se assim a importância de exames de imagens para investigar patologias mediastínicas em felinos que apresentem sinais clínicos inespecíficos, assim como descrito por Patnaik et al (2003). 
Síndromes paraneoplásicas como miastenia gravis, dermatite esfoliativa, eritema multiforme, hipercalcemia, linfocitose $\mathrm{T}$, anemia e polimiosite são frequentes em neoplasias tímicas e podem ser observadas no início ou no decorrer da doença (Day, 1997; Moffet, 2007; Zitz et al., 2008; Singh et al., 2010; Tepper et al. 2011; Kasabalis et al., 2011; Horta et al., 2018; Byas et al., 2019). Neste caso descrito não foram evidenciadas síndromes paraneoplásicas, porém a evolução do quadro para o óbito foi rápida. A presença de síndromes paraneoplásicas influencia diretamente no prognóstico como a classificação histológica e o estadiamento clínico (Cavalcante et al., 2014; Angelou et al., 2016; Horta et al., 2018).

\section{Conclusão}

Embora os timomas sejam neoplasias de ocorrência rara em felinos, eles podem ocorrer nesta espécie, com apresentação clínica inespecífica, sendo a necropsia e a histopatologia ferramentas importantes para a realização do diagnóstico. Além disso, a imo-histoquímica representa uma importante técnica para determinar o subtipo de timoma.

\section{Referências}

Angelou, V., Patsikas, M. N., Psalla, D. \& Papazoglou, L. G. (2016). Diagnosis and surgical treatment of thymoma in the cat. Hellenic Journal of Companion Animal Medicine, 5(1):61-68.

Bandarra, E. P., Sequeira, J. L., Moura, V. M. B. D. \& Ferreira, H. (2000). Timoma em cão: Relato de caso. Brazilian Journal of Veterinary Research and Animal Science, 37(5):400-404.

Byas, A. D., Applegate, T. J., Stuart, A., Byers, S. \& Frank, C. B. (2019). Exfoliative dermatitis associated with thymoma in goat: case report and brief literature review. Journal of Veterinary Diagnostic Investigation, 31(6): 905-908.

Capelozzi, V. L. (2009). Papel da imuno-histoquímica no diagnóstico do câncer de pulmão. Jornal Brasileiro de Pneumologia, 35(4): 375-382.

Cavalcanti, J. V., Moura, M. P. \& Monteiro, F. O. (2014). Thymoma associated with exfoliative dermatitis in a cat. Journal of Feline Medicine and Surgery, 16(12): 1020-1023.

Day, M. J. (1997). Review of thymic pathology in 30 cats and 36 dogs. Journal of Small Animal Practice, 38(9): $393-403$.

Detterbeck, F. \& Parsons, A. M. (2004). Thymic tumours. The Annals of Thoracic Surgery, 77(5): 1860-1869.

Freitas, R. R., Irino, E. T., Macruz, R., Simões, E. A., Monteiro, R., Aiello, V. D., Stopiglia, A. J. \& Jatene, F. B. (2002). Morte súbita por compressão de timolipoma em cão. Relato de caso. Ciência Animal, 12(2): 99-103.

Horta, R. S., Figueiredo, M. S., Costa, M. B. F., Costa, M. P., Silva, L. V., Gonçalves, A. B. C. \& Cassali, G. D. (2018). Timoma canino associado à miastenia gravis. Acta Scientiae Veterinariae, 46(1): 277.

Kasabalis, J. D., Mylonakis, M. E., Patsikas, M. N., Petanides, T. \& Koutinas, A. F. (2011). Paraneoplastic exfoliative erythroderma in a cat with thymoma. Journal of the Hellenic Veterinary Medical Society, 62: 229-234.

Moffet, A. C. (2007). Metastatic thymoma and acquired generalized myasthenia gravis in a beagle. Canadian Veterinary Journal, 48(1): 91-93.

Oliveira, T. E., Oliveira, P. P., Duarte, E. G., Otero, C. V. L., Araújo, V. J. \& Lima, B. T. A. R. (2021). Timoma em cão: Relato de caso. PUBVET, 15(05): 19.

Ortemberg, L. L., Fidanza, M. M., Pereira, M. \& Bartolomeo, M. (2015). Timoma en el gato. Reporte de un caso en el Hospital Escuela de la Facultad de Ciencias Veterinarias, UBA. Revista veterinaria argentina, 32(330): 1-6.

Patnaik, A. K., Lieberman, P. H., Erlandson, R. A. \& Antonescu, C. (2003). Feline cystic thymoma: a clinicopathologic, immunohistologic, and electron microscopic study of 14 cases. Journal of Feline Medicine and Surgery, 5: 27-35.

Souza, H. J. M., Amorim, F. V., Jaffé, E., Gorgozinho, K. B., Calixto, R. S., Silva, J.G. \& Evandro, T. P. (2005). Timoma e tumor de células da granulosa em gata. Acta Scientiae Veterinariae, 33(2): 211-217.

Singh, A., Boston, S. E. \& Poma, R. (2010). Thymoma-associated exfoliative dermatitis with post-thymectomy myasthenia gravis in a cat. Canadian Veterinary Journal, 51(7): 757-760.

Tepper, L. C., Spiegel, I. B. \& Davis, G. J. (2011). Diagnosis of erythema multiforme associated with thymoma in a dog and treated with thymectomy. Journal of the American Animal Hospital Association, 47(2): 19-25.

Valli, V. E., Bienzle, D. \& Meuten, D. J. (2017). Tumors of the Hemolymphatic System. In: Meuten D.J. Tumors in Domestic Animals. (5a ed.), California: John Wiley \& Sons, p.203-321. 
Research, Society and Development, v. 10, n. 8, e28110817384, 2021

(CC BY 4.0) | ISSN 2525-3409 | DOI: http://dx.doi.org/10.33448/rsd-v10i8.17384

Yoon, J., Feeney, D. A., Cronk, D. E., Anderson, K. L. \& Ziegler, L. E. (2004). Avaliação tomográfica computadorizada de massas mediastinais caninas e felinas em 14 pacientes. Veterinary Radiology \& Ultrasound, 45(6): 542-546.

Zitz, J. C., Birchar, D. S. J., Couto, G. C., SamiI, V. F., Weisbrode, S. E. \& Young, G. S. (2008). Results of excision of thymoma in cats and dogs: 20 cases (1984-2005). Journal of the American Veterinary Medical Association, 232(8): 1186-1192. 